\title{
THE REMODELING OF LEFT ATRIUM AND VASODILATION FACTORS CHANGES IN ISCHEMIC HEART FAILURE
}

\author{
V. I. Denesyuk, O. V. Denesyuk, N. O. Muzyka \\ NATIONAL PIROGOV MEMORIAL MEDICAL UNIVERSITY, VINNYTSIA, UKRAINE
}

Background. According to the national registries of European countries and epidemiological studies, the prevalence of chronic heart failure (CHF) among adults is 2,0-5,0\%, and increases due to age, in people aged over 70 years old it is 10,0-20,0\%.

Objective. To find out the specific features of remodeling of the left atrium and change of vasodilation factors in ischemic heart failure with reduced and preserved left ventricular ejection fraction and to establish correlation relationships.

Methods. A full clinical examination of 153 patients with CHF (105 men and 48 women) was conducted to achieve this objective. The surveyed patients underwent clinical examinations; spectrophotometric parameters: quantification of markers of vasodilation, metabolites of monoxide nitrogen - nitrates and nitrites with Gris reagent; content of endothelial nitric oxide synthase (eNOS) in serum - ELISA for the set of Nitric Oxide Synthase 3, Endothelial (NOS3) Human ELISA Kit (Cloud-Clone Corp, USA). Electrocardiographic (ECG) examination was conducted in 12 standard conventional leads on electrocardiograph by the Hungarian production Heart Screen 112 D.

Results. The $1^{\text {st }}$ group of the examined patients with reduced LV EF prevails III (significant) degree LA dilatation in 33 (70.21\%) cases, II (moderate) degree of LA dilatation was determined in 14 (29.78\%), and I (initial) degree was not defined at all. In the $2^{\text {nd }}$ group of the patients with preserved LV EF mainly the II degree of LA dilatation was determined in 44 (44.51\%) cases, and decreased LA dilation in 39 (36.79\%) cases ( $p<0.01)$, and III degree of LA dilation was defined in $23(21.69 \%)$ cases $(p<0.01)$. In patients with stable coronary heart disease, complicated by heart failure with reduced LV EF and II degree of LA dilatation, eNOS levels in the serum was $449.00 \pm 39.91 \mathrm{pg} / \mathrm{ml}$, whereas in patients with stable coronary heart disease, complicated by heart failure with preserved LV EF and II stage of LA dilatation $-673.56 \pm 50.98 \mathrm{pg} / \mathrm{ml}(p<0.01)$. At III stage of LA dilatation in patients of the $1^{\text {st }}$ group level eNOS was $344.20 \pm 51.98 \mathrm{pg} / \mathrm{ml}$ in the patients of the $2^{\text {nd }}$ group $-616.90 \pm 36.49 \mathrm{pg} / \mathrm{ml}(\mathrm{p}<0.01)$. At the same degree and with LA dilation in the patients of the $2^{\text {nd }}$ group eNOS was $750.27 \pm 99.85 \mathrm{pg} / \mathrm{ml}$.

Conclusions. The structural and functional changes of the left atrium and changing factors of vasodilation in patients with stable coronary artery disease of II-III functional classes complicated by heart failure of I-III functional classes are studied. It is established that in the examined patients with stable coronary heart disease complicated by heart failure with reduced left ventricular ejection fraction mainly III (significant) degree of dilatation of the left atrium was determined, while in patients with stable coronary heart disease, complicated by heart failure with preserved left ventricular ejection fraction mainly II (moderate) degree of dilation of the left atrium was determined. In comparison with the results of research among the patients with stable coronary heart disease, complicated by heart failure with reduced left ventricular ejection fraction, and a group of patients with preserved left ventricular ejection fraction, it was determined a significant decrease in eNOS, nitrites, total amount of nitrites and nitrates.

KEY WORDS: heart remodeling factors of vasodilation, heart failure.

\section{Introduction}

According to the national registries of European countries and epidemiological studies, prevalence of chronic heart failure (CHF) among adults is $2.0-5.0 \%$, and increases in proportion to age, people aged over 70 years it is from 10.0 to $20.0 \%$ [16]. Considering demo-

Corresponding author: Nadia Muzyka, Department of General Medicine № 3, National Pirogov Memorial Medical University, 96, Khmelnytske Chaussee Street, Vinnytsia, Ukraine, 21000 Phone number: +380673757410

E-mail: myzuchechka@gmail.com graphic trends, in Ukraine as well, to increase the number of population of older age groups, the provision of medical care to patients with $\mathrm{CHF}$ is becoming a topical issue $[2,14]$.

Distinguish systolic and diastolic options of $\mathrm{CHF}$. In version of systolic CHF the presence of clinical signs in patients with ejection fraction (EF) of $\mathrm{LV} \leq 45.0 \%$, at diastolic version with $\mathrm{LV}$ EF $>45.0 \%$ is observed [4]. It is known that left ventricular hypertrophy (LVH) precedes the development of $\mathrm{CHF}$ and is formed together 
with or even is caused by this disease. Analysis of prognostic value of LVH in patients with CHF with preserved ejection fraction proves that the high risk of lethal outcome is observed in patients with left ventricular myocardial mass that exceeds $400 \mathrm{~g}$ [3]. It is established that at the progression of diastolic dysfunction an increased risk of sudden cardiac death in $80.0 \%$ occurs $[4,15]$. Endothelial dysfunction is very important in development of CHF. The cause of nitric oxide (NO) synthesis inhibition by endothelium as a major component of its dysfunction at CHF is free radical stress [11]. The main cause of CHF is negative impact of free radical factors that leads to lower ventricular myocardial contractile function followed by entire body hypoxia, endothelial dysfunction with the appropriate activation of cellular factors [17]. These processes can lead to increasing of blood coagulation capacity, which is one of the causes of thrombotic complications in patients with CHF [12]. Biosynthesis of NO from L-arginine occurs in three major isoforms of NO-synthase, two constitutive: neuronal (nNOS) and endothelial (eNOS), and inducible one (iNOS) [6].

The objective of the study is to find out the specific features of left atrium remodeling and vasodilation factors changes in case of ischemic heart failure with reduced and preserved left ventricular ejection fraction and to establish correlation relationships.

\section{Material and Methods}

To achieve this objective a full clinical examination of 153 patients with CHF ( 105 men and 48 women) was conducted; they were treated in the Department of Cardiology for patients with arrhythmias of Vinnitsa Regional Centre of Cardiovascular Disorders (Ukraine). All patients were divided into 2 groups: group 1 consisted of patients with stable coronary heart disease complicated by heart failure with reduced LVEF $(\mathrm{n}=47)$; group 2 - patients with stable coronary heart disease complicated by heart failure with preserved LV EF $(n=106)$. The control group consisted of 30 healthy individuals. The diagnoses of $\mathrm{CH}$ and $\mathrm{FC}$ was made due to clinical, laboratory and instrumental criteria, as recommended by the European Society of Cardiology (2012) and the Association of Cardiologists of Ukraine (2012) [16]. Diagnosis and treatment of clinical forms of coronary artery disease were made by order of the Ministry of Health of Ukraine № 152 from 03.02.2016 [9]; functional class (FC) $\mathrm{CH}$ - by NYHA classification.
The criteria for inclusion. Patients with stable coronary artery disease II-III FC followed by CHF II-III FC on informed consent to participate in the study. Exclusion criteria: CHF FC IV by the NYHA; period up to 3 months from the beginning of acute myocardial infarction, or stroke; implanted artificial pacemaker or need of implantation; severe diseases of respiratory system, kidneys, liver with hepatic insufficiency, anaemic state of haemoglobin $90,0 \mathrm{~g} / \mathrm{dL}$ and below; the formation of malignant disorders and severe neuro-psychiatric disorders.

Among the patients who participated in the study mail prevailed. The average age of patients was $68.80 \pm 0.90$ years. Hypertension was defined in 132 (86.27\%) patients. Systolic blood pressure (BP) was $142.40 \pm 5.01 \mathrm{~mm} \mathrm{Hg}$, diastolic blood pressure $-88.30 \pm 2.95 \mathrm{mmHg}$

The examined patients underwent clinical (complaints, case history, physical examination); laboratory tests (general clinical blood and urine tests, blood glucose, bilirubin, cholesterol, $\beta$-lipoprotein, creatinine, urea, coagulation parameters); spectrophotometric parameters: quantification of vasodilation markers metabolites of monoxide nitrogen: nitrates and nitrites with Gris reagent; content of endothelial nitric oxide synthase (eNOS) in serum - ELISA for the set of Nitric Oxide Synthase 3, Endothelial (NOS3) Human ELISA Kit (Cloud-Clone Corp, USA). Electrocardiographic (ECG) examination was conducted in 12 standard conventional leads on electrocardiograph Heart Screen 112 D made in Hungary. Statistical analysis was performed with standard statistical package STATISTICA 6.0. For primary analysis of tables and intermediate calculations Microsoft Excel package was used.

\section{Results and Discussion}

The decrease in left atrium (LA) dilation degree in patients with stable coronary heart disease complicated by heart failure with reduced and preserved LV EF is presented in Table 1.

In Table 1 the $1^{\text {st }}$ group of the examined patients with reduced LV EF prevails III (significant) degree of LA dilatation in 33 (70.21\%) cases, II (moderate) degree of LA dilatation in $14(29.78 \%)$, I (initial) degree is not defined at all. In the $2^{\text {nd }}$ group of patients with preserved LV EF the degree of dilatation II LA was determined in $44(44.51 \%)$ cases, and slightly decreased degree of LA dilation in 39 (36.79\%) cases $(p<0,01)$, III degree of LA dilation in 23 $(21,69 \%)$ cases $(p<0,01)$. 
Table 1. Remodeling of left atrium in case of stable coronary heart disease complicated by heart failure with reduced and preserved left ventricular ejection fraction

\begin{tabular}{|l|c|c|c|}
\hline The degree of dilation LA & $\begin{array}{c}\text { Patients with heart failure } \\
\text { with reduced LV EF group 1, } \\
\mathrm{n}=47\end{array}$ & $\begin{array}{c}\text { Patients with heart failure } \\
\text { with preserved LV EF group 2, } \\
\mathrm{n}=106\end{array}$ & $\mathrm{p}$ \\
\hline $\begin{array}{l}\text { I (initial) LA from } \\
40.0 \text { to } 45.0 \mathrm{~mm}\end{array}$ & 0 & $39(36.79 \%)$ & $\mathrm{p}<0,01$ \\
\hline $\begin{array}{l}\text { II (moderate) LA from } \\
45.1 \mathrm{~mm} \text { to 50.0 mm }\end{array}$ & $14(29.78 \%)$ & $44(41.51 \%)^{*}$ & $\mathrm{p}<0,01$ \\
\hline $\begin{array}{l}\text { III (significant) LA from } \\
50.1 \mathrm{~mm} \text { and more }\end{array}$ & $33(70.21 \%)^{*}$ & $23(21.69 \%)$ & $\mathrm{p}<0,01$ \\
\hline
\end{tabular}

Notes: LA - the size of the left atrium; * $p<0.01$ - reliable change index.

Remodeling of myocardium, including hypertrophy and dilation of left ventricle with a changes of its geometry and a contraction violation precedes clinical skim CHF manifestations [7]. Thus, remodeling is a harbinger of heard activity decompensation $[7,13]$. The features of myocardial remodeling are studied the most in patients after myocardial infarction with heart failure development $[7,8,14]$.

These results are consistent with the data that myocardial ischemia may be accompanied by its dysfunction [7].

The study of factors of vasodilation levels and degrees of LA dilatation in patients with stable coronary heart disease complicated by heart failure with reduced and preserved LV EF is presented in Table 2.

In patients with stable coronary heart disease complicated by heart failure with reduced LV EF and II degree of LA dilatation, eNOS levels in the serum is $449.00 \pm 39.91 \mathrm{pg} / \mathrm{ml}$, whereas in patients with stable coronary heart disease complicated by heart failure with preserved LV EF and II stage of LA dilatation -
$673.56 \pm 50.98 \mathrm{pg} / \mathrm{ml}(\mathrm{p}<0.01)$. At III stage of LA dilatation in patients of group 1 the eNOS level is $344.20 \pm 51.98 \mathrm{pg} / \mathrm{ml}$ in patients of groups $2-616.90 \pm 36.49 \mathrm{pg} / \mathrm{ml}(\mathrm{p}<0,01)$. At the same degree and with LA dilation in patients of group 2 eNOS is $750.27 \pm 99.85 \mathrm{pg} / \mathrm{ml}$. Thus, in the examined patients with the progression of heart failure the endothelial factors decrease, e.g. eNOS.

In the study of levels of metabolites of nitrogen monoxide - nitrites and nitrates in blood serum the following data were obtained: in patients with stable coronary heart disease, complicated by heart failure with reduced LV EF and II and III stage of LA dilatation, the total level of nitrates and nitrites in serum was significantly lower than in patients with stable coronary heart disease, complicated by heart failure with preserved LVEF and II and III stage of $L A$ dilatation $(p<0.01)$.

Among the patients of group 1 with reduced LVEF with the progression of LA dilation degree from II (moderate) stage to III (large) one, eNOS levels in blood serum decreased by $23.34 \%$.

Table 2. Vazodilating factors at different stages of left atrium dilation in case of stable coronary heart disease complicated by heart failure with reduced and preserved left ventricular ejection fraction $(\mathrm{M} \pm \mathrm{m})$

\begin{tabular}{|l|c|c|c|c|c|}
\hline \multirow{2}{*}{ Indices } & \multicolumn{2}{|c|}{$\begin{array}{c}\text { Patients with heart failure with } \\
\text { reduced LV EF group 1, } \mathrm{n}=47\end{array}$} & \multicolumn{3}{|c|}{ Patients with heart failure with preserved LV EF } \\
\cline { 2 - 6 } & $\begin{array}{c}\text { II degree of LA } \\
\text { dilatation } \\
\mathrm{n}=14\end{array}$ & $\begin{array}{c}\text { III degree of LA } \\
\text { dilatation } \\
\mathrm{n}=33\end{array}$ & $\begin{array}{c}\text { I degree of LA } \\
\text { dilation } \\
\mathrm{n}=39\end{array}$ & $\begin{array}{c}\text { II degree of LA } \\
\text { dilatation } \\
\mathrm{n}=44\end{array}$ & $\begin{array}{c}\text { III degree of LA } \\
\text { dilatation } \\
\mathrm{n}=23\end{array}$ \\
\hline eNOS, $\mathrm{pg} / \mathrm{ml}$ & $449.00 \pm 39.91$ & $344.20 \pm 51.98$ & $750.27 \pm 99.85$ & $673.56 \pm 50.98^{*}$ & $616.90 \pm 36.49^{\#}$ \\
\hline $\begin{array}{l}\text { Nitrite+nitrate, } \\
\mathrm{mmol} / \mathrm{l}\end{array}$ & $16.75 \pm 0.75$ & $16.05 \pm 0.94$ & $19.27 \pm 0.67$ & $19.22 \pm 0.77^{*}$ & $19.05 \pm 1.10^{\#}$ \\
\hline Nitrite, $\mathrm{mmol} / \mathrm{l}$ & $4.41 \pm 0.21$ & $3.95 \pm 0.32$ & $5.15 \pm 0.20$ & $5.06 \pm 0.16^{*}$ & $5.30 \pm 0.37^{\#}$ \\
\hline Nitrate, $\mathrm{mmol} / \mathrm{l}$ & $12.34 \pm 0.59$ & $12.09 \pm 1.13$ & $14.11 \pm 0.61$ & $14.16 \pm 0.71 *$ & $13.74 \pm 0.85$ \\
\hline
\end{tabular}

Notes:

* - comparison of patients with stable coronary heart disease complicated by heart failure with reduced LV EF and preserved LV EF and II degree of left atrium dilatation $(p<0,01)$;

\# - comparison of patients with stable coronary heart disease complicated by heart failure with reduced LV EF and preserved LV EF and III degree of left atrium dilatation $(p<0,01)$. 
Among the patients of group 2 with preserved LVEF with the progression of LA dilatation from I (initial) stage to II one, the level of eNOS in blood serum decreased by $12.89 \%$, and with the progression of the II stage to III one LA dilation reduced by $8.41 \%$.

In the examined patients with stable coronary artery disease complicated by heart failure with reduced left ventricular ejection fraction, the correlation analysis between indicators of linear dimensions of LA and eNOS levels in the blood serum a direct correlation of medium strength $(r=0.38)(p<0,01)$ was established. Carrying out a similar correlation analysis for patients with stable coronary heart disease complicated by heart failure with preserved LV EF also allowed to establish a direct correlation of weak force $(r=0.21)(p<0.01)$.

Progressive hypertrophy and dilatation of heart was followed by subsequent first diastolic disruption and then systolic ventricular dysfunction, increased myocardial oxygen demand, changes in subendocardial blood flow, myocardial bioenergy disorders and increased risk of life-threatening ventricular arrhythmias [1].

Recent research proved instrumental and morphological parallels, so we expand on the remodeling of heart as a single clinical and morphological syndrome that is important for making prognosis in patients with coronary heart disease [18].

\section{References}

1. Amosowa K. Clinical Cardiology 1999: 710.

2. Voronkov L. Primary prevention of heart failure - one of the priorities of modern cardiology. Ukrainian kardiology magazine 2004; 4: 42-47.

3. Glaser M. Left ventricular hypertrophy and prognosis in patients with chronic heart failure with preserved ejection fraction. Heart Failure 2012; 13: 19-25.

4. Denesyuk O, Denesyuk V. Dynamics degrees of remodeling and systolic-diastolic left ventricular dysfunction in unstable angina influenced by the optimal long-term treatment. Herald of problems of
The processes of LA remodeling are defined by the degree of new myocardial damage and the presence of viable myocardium.

\section{Conclusions}

In patients with stable coronary heart disease complicated by heart failure with reduced left ventricular ejection fraction III (significant) degree of left atrium dilatation was mainly determined, while in patients with stable coronary heart disease complicated by heart failure with preserved left ventricular ejection fraction II (moderate) degree of left atrium dilation was mainly determined.

In the patients with stable coronary heart disease complicated by heart failure with reduced left ventricular ejection fraction in comparison with the group of patients with preserved left ventricular ejection fraction, the significant reduction of eNOS, nitrites, total of nitrites and nitrates was defined during the study.

The research allowed to establish the direct correlation of medium strength $(r=0.38)$ between parameters of linear left atrial size and eNOS levels in blood serum of the patients with stable coronary artery disease complicated by heart failure with reduced left ventricular ejection fraction $(p<0.01)$ and the direct correlation of weak force $(r=0.21)$ in the examined patients with stable coronary artery disease complicated by heart failure with preserved left ventricular ejection fraction $(p<0,01)$.

biology and medicine 2015; 2: 121-126.

5. Dyuzhykov A, Terentyev V. Features of morpho-functional state of the myocardium in patients with ischemic and no ischemic cardiomyopathy. Modern scientific technology 2007; 12: 23-28.

6. Zharynova V. Modern Opportunities of optimization of endothelium treading therapy in patients with CHF (focus on eNOS). Heart failure 2013; 3: 46-53.

7. Zakirova AN, Oganov RG, Zakirova NE, Klochkova GR, Musina FS. Myocardial remodeling in ischemic heart disease. Rational Pharmacotherapy in Car- 
diology 2009; 5(1): 42-45. (In Russ.) DOI:10.20996/18196446-2009-5-1-42-45.

8. Lang R, Biering M, Devereux RB et al. Recommendations of chambers quantification. Eur J Echocardiogr 2006; 7(2): 79-108.

9. Mandate of the Ministry of Health of Ukraine from 02.03.2016 №152 On approval of the introduction of medical and technological documents for standardization of care in stable coronary heart disease. News of medicine and pharmacology in Ukraine 2016; 572: 26-60.

10. Muhorlyamov N. Cardiomyopathy. Medicine 1999: 228.

11. Oxidative stress in chronic heart failure. The possibilities of pharmacological correction. Y Belenkov, E Privalov, Y Danilogorskaya et al. Cardiology. Cardiovascular Surgery 2009; 1: 4-9.

12. Patalakh I. Participation of protein $C$ in the regulation of the fibrinolytic capacity of plasma in patients with chronic heart failure. Circulation of blood and hemostasis 2009; 3/4: 95-100.

13. Pfeffer MA, Braunwald E. Ventricular remodeling after myocardial infarction. Experimental observations and clinical implication. Circulation 1990; 81(4):1161-1172.

14. Recommendations of Association of Cardiologists of Ukraine for the treatment of chronic heart failure in adults (revision 2011). Heart Failure 2011; 1: 101-116.

15. Aljaroudi W. Inpact of progression of diastolic dysfunction on mortality in patients with normal ejection fraction. Circulation 2012; 14: 782-788.

16. ESC Guidelines for the diagnosis and treatment of acute and chronic heart failure 2012: The Task Force for the Diagnosis and Treatment of Acute and Chronic Heart Failure 2012 of the European Society of Cardiology. Developed in collaboration with the Heart Failure association (HFA) of the ESC. European Heart Journal 2012; 33: 1718-1847.

17. Ramani GV. Chronic Heart Failure: Contemporary Diagnosis and Management. Mayo Clin. Proc. 2010; 85: 180-195.

18. Ziuraitiene R, Pangonyte D, Stalioraityte E et al. Cardiomyocyte remodeling in ischemic heart disease. Medicina (Kaunas) 2008; 44: 848-854. 